\title{
Signal Processing Algorithm to Reduce RWR Electro- Magnetic Interference with Tail Rotor Blade of Helicopter
}

\author{
Hyo-Bin Im*, Eun-Kyoung Go*, Un-Seob Jeong* and Si-Chan Lyu* \\ Agency for Defense Development, Daejeon, Korea, 305-600
}

\begin{abstract}
In the environment where various and complicated threat signals exist, RWR (Radar Warning Receiver), which can warn pilot of the existence of threats, has long been a necessary electronic warfare (EW) system to improve survivability of aircraft. The angle of arrival (AOA) information, the most reliable sorting parameter in the RWR, is measured by means of four-quadrant amplitude comparison direction finding (DF) technique. Each of four antennas (usually spiral antenna) of DF unit covers one of four quadrant zones, with 90 degrees apart with nearby antenna. According to the location of antenna installed in helicopter, RWR is subject to signal loss and interference by helicopter body and structures including tail bumper, rotor blade, and so on, causing a difficulty of detecting hostile emitters. In this paper, the performance degradation caused by signal interference by tail rotor blades has been estimated by measuring amplitude video signals into which RWR converts RF signals in case a part of antenna is screened by real tail rotor blade in anechoic chamber. The results show that corruption of pulse amplitude (PA) is main cause of DF error. We have proposed two algorithms for resolving the interference by tail rotor blades as below: First, expand the AOA group range for pulse grouping at the first signal analysis phase. Second, merge each of pulse trains with the other, that signal parameter except PRI and AOA is similar, after the first signal analysis phase. The presented method makes it possible to use RWR by reducing interference caused by blade screening in case antenna is screened by tail rotor blades.
\end{abstract}

Key words : RWR, Radar Warning Receiver, Tail Rotor Blade, Signal Interference

\section{Introduction}

EW (Electronic Warfare) environment is getting complex persistently. RWR to protect aircraft from various radar threats, is essential gradually.

But, in rotary-wing aircraft, RF signal interference by air-frame occurs greater than fixed-wing aircraft due to limited install requirements. By this reasons, some problems occur in RWR such as DF for radar signal source and signal characteristic analysis.

To mount antennas where interferences do not occur will be ideal to solve these problems, but practically it is impossible due to restriction of air-frame design, and other conditions. So, the additional processing in RWR is needed to solve these problems.

* Researcher

E-mail : anoint@add.re.kr

Tel : +82-42-821-2995

Fax : $+82-42-821-3400$ 
Factors causing signal interference, are very various like main rotor and tail rotor, aircraft body, landing gear, weapons and other equipment installed on the outside of helicopter. The signal interference occurs more severely in an antenna installed on tailboom than in an antenna installed on nose-bay due to structural characteristic of helicopter. Because it is difficult to secure FOV (Field of view) due to outside structures that exist on the back of helicopter such as tail rotor blade, vertical/horizontal pin stabilizer, tail bumper, other structures, etc. This phenomenon can be explained by signal reflections and attenuations or diffractions. In this case, the FAR(False Alarm Rate) increases by deteriorate of signal analysis and identification accuracy.

In an aspect of integrated electronic warfare, it is most important factor to detect radar signal accurately. The information made from RWR is the key point for identification and counter-measuring of threat. More accurate information leads more accurate and efficient counter-measuring. And finally it can increase the survivability of aircraft. By these reasons, reducing the electro-magnetic interference of tail-rotor blades is essential.

In this paper, results of experiment using real rotor blades about signal interference by tail rotor blade which is the major factor causing signal attenuation and analysis result are shown. Finally the resolving algorithms are proposed.

\section{Signal Interference by Tail Rotor Blade}

\subsection{Signal processing flow of the RWR}

Signal processing flows of RWR are divided into two parts. One is the signal reception part and the other is the analyzing part of received signal. The SRU (Signal Receiving Unit) receives the RF signal and send both video signal and frequency signal to the SAU (Signal Analysis Unit). The SAU generates PDW (Pulse Description Word) datas for each received pulses and extracts pulses that have same characteristics. And then makes pulses to pulsetrains. In this phase, the processes are performed as PRI(Pulse Repetition Interval) deinterleaving and estimation of the AOA (Angle of Arrival) by calculating PA (Pulse Amplitude) difference of each pulses. The AOA estimation is archived by comparison PA of each antenna. And then find the max channel that most strong signal received, and the second max channel. Then calculates PA difference with each other, and matches it to the AOA table. As a result of this procedure, we can decide the AOA of the signal. After this, compare analyzed pulse-train data as a result of these processes with embedded signal parameter library, and identify the threats which emit the signals.

The locations of mounted RWR antennas are depicted in Fig. 1.

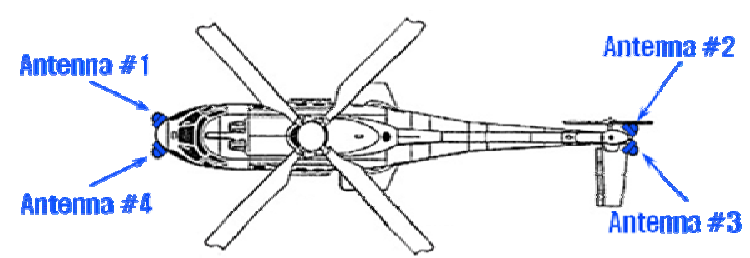

Fig. 1. Locations of RWR antennas

\subsection{Effects of blade reflections}

Tail rotor blade of helicopter is made of a composite of nickel, titanium, urethane, etc., in steel-frame structure generally. Outer surface is covered by copper mesh to prevent damages from thunderbolt. 
Analysis processes follow as: First, it is a signal reflection effect by blade. When both a direct signal and blade-reflected one reaches to antennas simultaneously, PRI de-interleaving is performed incorrectly and PA is changed also. As this result, accuracy of direction finding deteriorates finally.

Second, it is a direct PA attenuation of signals due to the reflections by tail rotor blade. Because tail rotor blade screens the receiving antenna during rotation, PA of signals received to antenna is attenuated, and beam-pattern is distorted. Tail rotor blade's RPM(Rounds Per Minute) is variable to $90 \% \sim 110 \%$ of average RPM in proportion to RPM of main rotor. Therefore the estimation for periodical signal interference is difficult. In this way, PA of signal attenuated due to blade is reduced compared with normally received one. Therefore DF error is increasing greatly at RWR using DF technique by PA difference. If signal attenuation effect is big, it has influence on PRI de-interleaving process and finally, analysis result becomes inaccurate. And in phase of pulse-train extractions, single threat pulse train can be separated as more than two threat signals.

\section{Measurement \& Estimation of Signal Interference}

\subsection{Measurement of Signal Interference}

The test for measuring signal interference by blade was performed using real tail rotor blade of helicopter. Because the test is just for the measurement of the PA and AOA distortion pattern by blade, it might be different with real situation. The simplified test setup is depicted in Fig. 2.

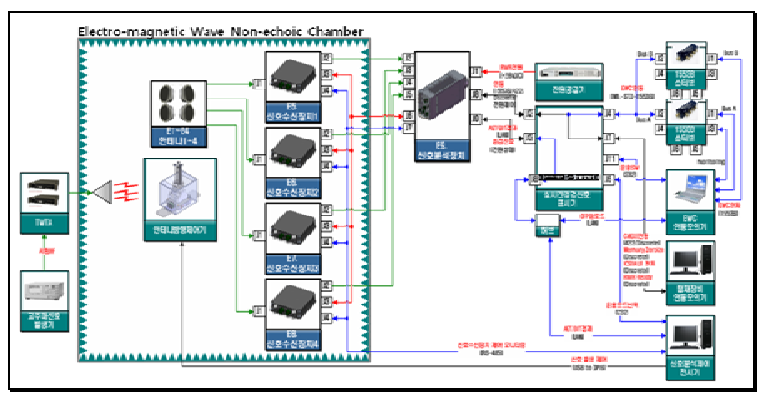

Fig. 2. Simplified test setup

For this test, the first step is to make a test signal parameter table that includes various signal characteristics such as frequency, frequency type, PRI, PRI type, PW (Pulse Width), etc. and the second step is to compose the test signal parameters that includes $\mathrm{min} / \mathrm{max}$ boundary values, which is representative value of each characteristic. The test is performed with $1 \mathrm{GHz}$ step within frequency band.

The next step is to repeat the test mentioned above when the tail rotor blade is not screened the receiving antenna, when screened about 50\%, when screened entirely, and when tail rotor blade is moving.

Interference measurements were finished by gathering signal waveforms and analysis result on SAU for each test case.

\subsection{Analysis of test result}

As a result of the test, the variation of signal interference pattern is not big and almost similar by variations of signal parameters such as frequency, frequency modulation, PRI, PRI type, PW, etc. 
As expected, signal interference is the least when blade is not screened the antenna, and when screened $50 \%$ is the next, and when screened entirely is the most serious.

On the other hand, against expectation, when blade is moving, the signal interference is bigger than when the antenna is screened by fixed blade. This phenomenon is caused not only by signal attenuation due to blade but also by signal diffraction and reflection by blade' s movement.

By the result of this test, the most severely influenced paramater is PA. And the others are nearly influenced. When severely influenced, the measurement shows that the attenuation of PA is about $5 \sim 6 \mathrm{~dB}$ less than not influenced. As a result of attenuation of PA, $\mathrm{DF}$ accuracy got worsen to the values of about $35^{\circ} \sim 50^{\circ}$. It is very serious degradation in the view of RWR performance. Please refer to Fig. $5 \sim$ Fig. 8 for the analysis of DF error.
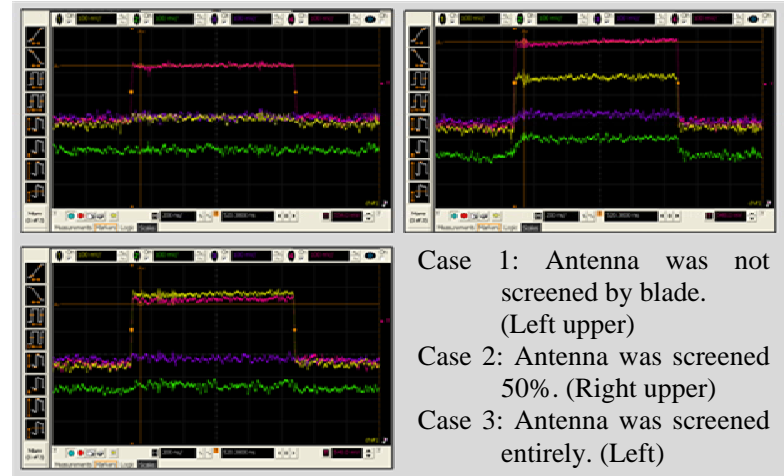

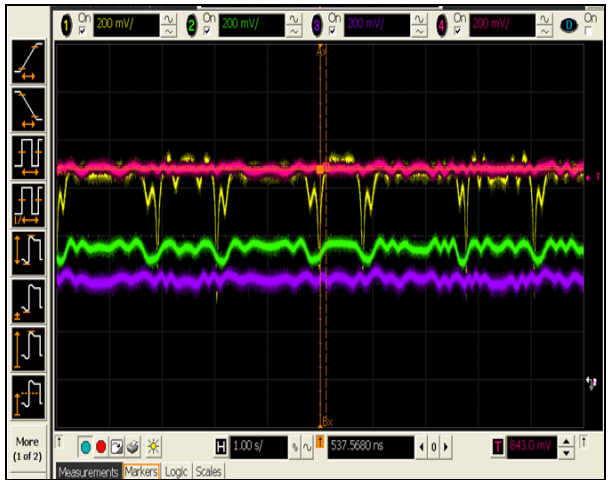

Fig. 4. Signal interference when blades moving

Fig. 3. Signal attenuation as blade position

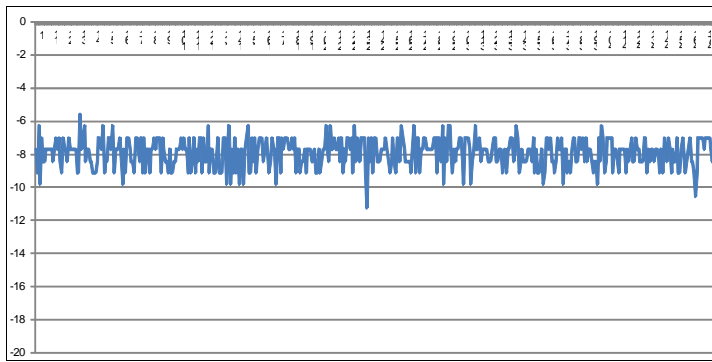

Fig. 5. DF error when antenna has no interference

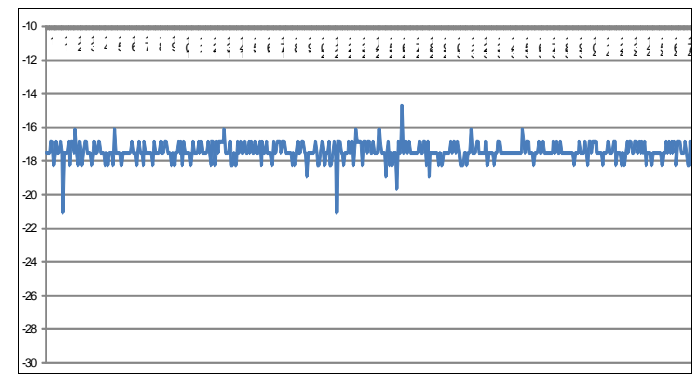

Fig. 6. DF error when antenna was screened

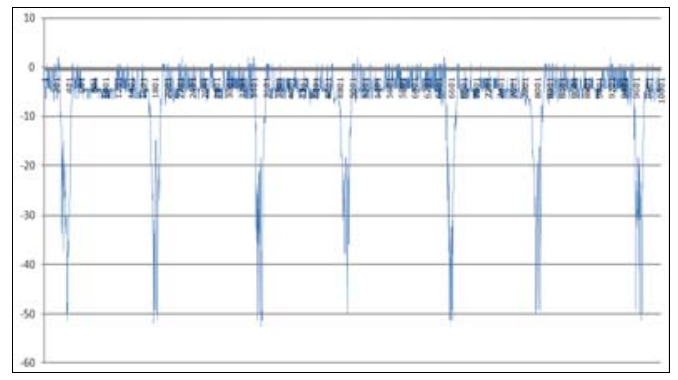

Fig. 7. DF error when antenna was screened entirely 


\subsection{Estimation of abnormal section by blade.}

Azimuth could be divided into 4 sectors according to the locations of emitters. (As results of Ch. 3.2, we know PA is varied by tail rotor blade, there is no increase of PA, just attenuation is occurred.)

First, consider the case when an emitter exists between $-180^{\circ}$ and $45^{\circ}$. This is a section where the blade interference does not exist. If an emitter exists between $270^{\circ}$ and $360^{\circ}$, the max channel is \#4 and the second max channel is \#1 or \#3, therefore this case is not influenced by blades. Next, if an emitter exists between $0^{\circ}$ and $45^{\circ}$, the max channel is \#1 and the second max channel is \#4, therefore this case is not influenced by blades too. And next, if an emitter exists between $225^{\circ}$ and $270^{\circ}$, the max channel is \#3 and the second max channel is \#4, therefore this is also not influenced by blade. Finally if an emitter exists between $180^{\circ}$ and $225^{\circ}$, the max channel is \#3 and the second max channel is \#2. In this case, although the second max channel is \#2, but because of signal arrived from the other side, AOA of the signal is not influenced by blade.

Second, consider the case when an emitter exists between $+45^{\circ}$ and $+90^{\circ}$. In this case, the estimated $\mathrm{AOA}$ exists not between $45^{\circ}$ and $90^{\circ}$ but between $0^{\circ}$ and $90^{\circ}$. Because the max channel is \#1 and the second max channel is \#2 normally, but PA of \#2 is attenuated by blades and then the third max \#4 becomes the second max channel.

Third, consider the case when an emitter exists between $+90^{\circ}$ and $+135^{\circ}$. In this case, the max channel is \#2 and the second max channel is \#1. But, if \#2 is attenuated by tail rotor blade and its PA becomes smaller than \#1, the second max channel, become the max channel and \#2 become the second max channel. Because of this, the AOA is between $45^{\circ}$ and $90^{\circ}$ instead of between $90^{\circ}$ and $135^{\circ}$.

Finally, consider the case when an emitter exists between $+135^{\circ}$ and $180^{\circ}$. The measured $\mathrm{AOA}$ exist between $135^{\circ}$ and $225^{\circ}$. In this case, the max channel is $\# 2$ and the second max channel is \#3. But as above case, when \#2 is attenuated by bladed, its PA becomes smaller than \#3 (the second max channel). \#3 becomes the max channel and \#2 becomes the second max channel. Because of this the AOA is between $135^{\circ}$ and $225^{\circ}$.

So, we synthesize the 4 cases described above, we can divide the azimuthal area into 3 sections. The first is reliable section that is never interfered by blade, the second is abnormal section \#1 that is interfered by blade when the max channel is antenna \#1, and the third is abnormal section \#3 that is interfered by blade when the max channel is antenna \#2.

According this assumption, we can draw the abnormal section like Fig. 9. The abnormal section \#1 and \#2 are AOA changed in each section, but do not depart with different section.

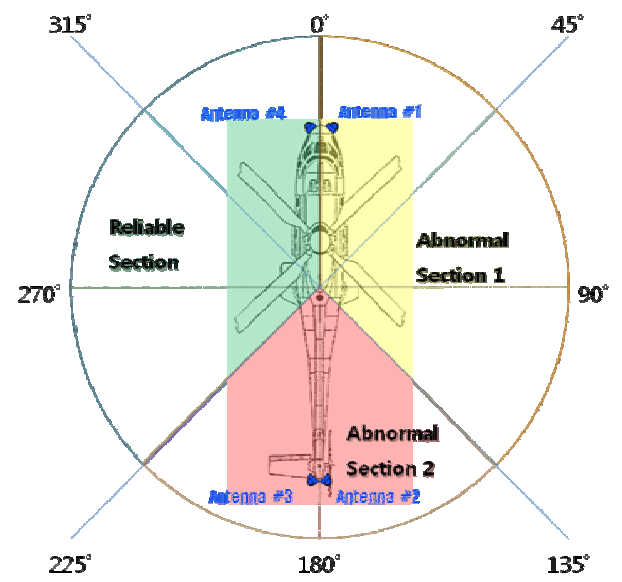

Fig. 9. The abnormal sections by tail rotor blade 


\section{Process for Interference Reduction}

General signal analysis procedures for RWR are as follows: pulse grouping for every received pulse, then extraction and merging pulse-trains (de-interleaving), and then verifying each pulse-trains. Fig. 10 shows the simplified signal analysis procedures of RWR.

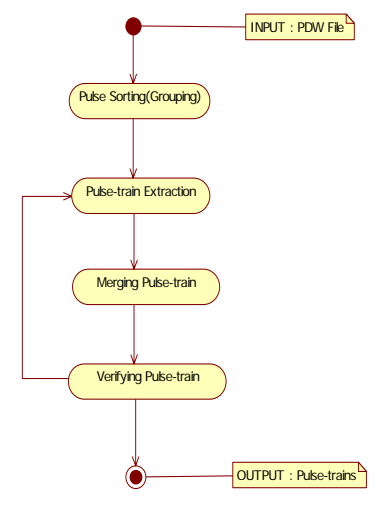

Fig. 10. Signal analysis process of RWR

\subsection{Problem of normal signal analyzing caused by tail rotor blade.}

As shown on Fig. 10, when performing a pulse grouping process before de-interleaving according to a specific parameter such as AOA, some pulses can be erroneously grouped to incorrect pulse groups by interferences caused by blades. As a result of this, some pulses are analyzed to more than two pulse-trains. If AOA difference between each pulse-train is smaller than system error, the pulse-trains are merged to one pulse-train. But if the AOA difference is bigger than system error, the pulse-trains remains to more than two.

The test using signal analysis algorithm on $\mathrm{PC}$-based platform was conducted to verify. For this test, we make simulated PDW data where only AOA parameter changed. Such as a result of the test using blades, the AOA variation is max $40^{\circ}$ and the shape of AOA variation set as a curve of secondary degree is shown in Fig. 11.

As a result of this test, 5 pulse-trains composed of 3 normal pulse-trains and 2 influenced pulse-trains, are extracted at the first phase as shown on Fig. 12. After the second phase, pulse-train merging, pulse-trains which have only the different duration time are merged with each other. Finally the analyzing results, which have different AOA, are produced.

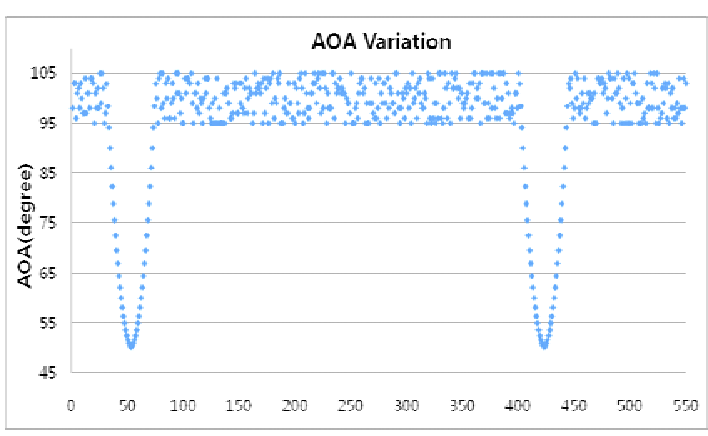

Fig. 11. AOA variation of test signals
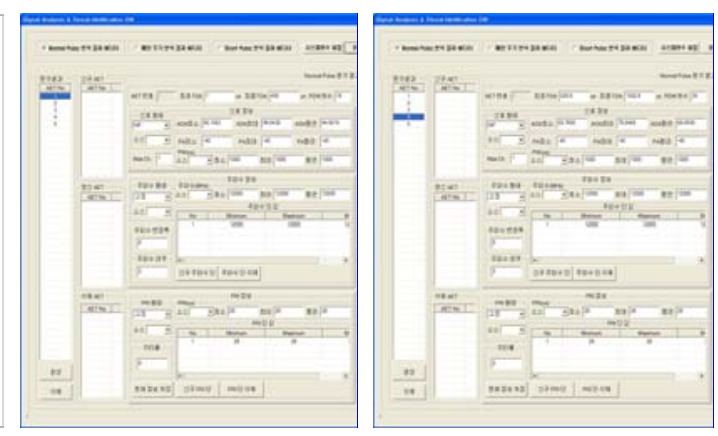

Fig. 12. An example of analysis result for normal pulse-trains (Left) \& for abnormal pulse-trains(Right) 


\subsection{Process of interference reduction using the extended AOA grouping algorithm}

AOA grouping is performed by making a histogram on the basis of the AOA of every pulse. Find the AOA Rank $(\mathrm{P})$ that its degree (number of pulses) is bigger than given (constant) value, and bind within specific range $(k)$ to one group. Therefore if the AOA of the pulses varied more than $\mathrm{k}$ value, the pulses cannot be same group with others and is departed to another group. And then, the extraction of pulse-train is conducted within each group.

$$
G_{\text {aоa }}=\left\{x \mid\left((P-k) \leq x_{\text {aоa }}\right) \text { and }\left(x_{\text {aоa }} \leq(P+k)\right)\right\},
$$

$$
\begin{aligned}
& \text { G : Group } \\
& x: \text { PDW } \\
& \text { P : AOA of Peak } \\
& k: \text { Standard value as same group }
\end{aligned}
$$

In the extended AOA grouping algorithm, check the AOA value when grouping, and if it is in the abnormal section by blade, searching pulses from existing norm to extended range. But search extended range until PDW that has continuous AOA value to minimize wrong grouping.

The AOA varied continuously by movement of blades, as shown the AOA variation pattern on Fig. 5. The signal processing procedure of proposed algorithm is as below.

First, create a group base on peak on the histogram same as former algorithm. Then conduct additional searching from $k$ to $k^{\prime}$. If find a rank that has zero degree while searching, then stop searching.

Emitters using similar signal more than one can exist actually, in multi-emitter situation. $k^{\prime}$ is experimental value and could be revised through more test.

$$
\begin{aligned}
& G_{\text {аоа }}^{\prime}=G_{\text {аоа }} \cup g_{\text {аоа }} \\
& g_{\text {аоа }}=\left\{x \mid\left(\left(P-k_{1}^{\prime}\right) \leq x_{\text {аоа }}\right) \text { and }\left(x_{\text {аоа }} \leq\left(P+k_{2}^{\prime}\right)\right)\right\}
\end{aligned},
$$

$g:$ additional member group

$k^{\prime}$ : extended range

(On condition that if there is zero degree of rank between $k$ to $k^{\prime}, k_{1}^{\prime}$ and $k_{2}^{\prime}$ become a rank as zero degree.)

As result of the test that applied the extended AOA grouping algorithm, all pulses are bound into one group, and analyzed into one threat signal.

Pulse grouping by the AOA is a kind of pre-processing procedure for enhancement of speed for de-interleaving. But in case that interference by blade exists, the reliability of the AOA grouping becomes worse. It is better not to use pulse grouping in that case. It is needed to use the existing AOA grouping algorithm and to use the extended AOA grouping algorithm only in abnormal section.

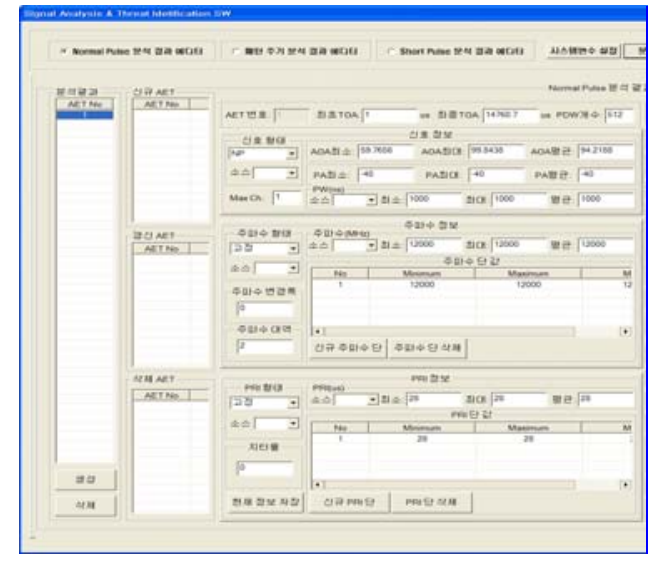

Fig. 13. Analyzed result in case of applied the extended AOA grouping algorithm 


\section{Conclusion}

In this paper, introduce the signal interference occurred to RWR antenna that installed on rotary-wing aircraft, and the test and measurement of the signal interference using real tail rotor blade of helicopter.

And the algorithm was suggested to resolve the signal interference and its performance was tested. The proposed algorithm can be applied to RWR so as to minimize DF error which is caused by tail rotor blade interference.

In this paper this test is not conducted in real situation that RWR is installed on target helicopter. In real situation the interference might be smaller than expected. It is necessary to conduct the test for worst-case and verify whether influenced pulse is processed successfully by using proposed algorithm. To get more satisfied test result, it is needed to conduct more tests in multi-emitter situation because missing pulse or similar signal can exist at the same time in multi-emitter situation.

It is difficult to get perfect solution for signal interference by blade because signal interference could be varied according to many reasons such as materials of blade, number of rotation per minute, installing location of antenna, etc.

As further study, it is better to classify the AOA variation pattern and re-calculate the AOA dynamically for enhancement of performance and accuracy of the algorithm.

And if the number of rotation per minute of tail rotor blade from aircraft system is known in advance, it will be possible to estimate more accurate, and to process for signal interference.

\section{Acknowledgment}

This paper is supported by the fund of KUH projects, DAPA, KOREA.

\section{References}

1. Richard G. Wiley, "ELINT:The Interception and Analysis of Radar Signals", ARTECH HOUSE, pp. 281-315

2. Chen. Victor C, "Radar signatures of rotor blades", Proc. SPIE, Vol. 4391, pp. 63$70,2001$. 